\title{
Movements induced by straight leg raising in the lumbo-sacral roots, nerves and plexus, and in the intrapelvic section of the sciatic nerve
}

\author{
M. D. GODDARD AND J. D. REID \\ From the Pathology Department, Wellington Hospital, Wellington, New Zealand
}

It is well known that on straight leg raising (Lasegue's test) traction is exerted on the sciatic nerve, the lumbo-sacral nerve roots, and the dura. The roots move through their intervertebral foramina for distances which may amount to several millimetres. If, however, dissection is made from the front instead of from the back (which is the usual approach) the course of the nerves and their changes with leg movements can be followed to the sciatic notch. This more extensive examination reveals several points not generally appreciated in descriptions of the sciatic nerve or in consideration of the significance of the straight-leg-raising test. In particular, it shows that nerve movements become progressively greater with increasing distance from the intervertebral foramina, and it establishes the existence of multiple points of pressure against the bone. Alterations in tension and the degree to which the roots are adherent to the intervertebral foramina are open to inspection. All of these changes can readily be examined and correlated with age.

This paper incorporates the objective findings and the conclusions reached after a considerable number of dissections made by an anterior approach after routine necropsy, and it records measurements of the movements of those nerves commonly implicated in sciatic pain in a smaller series of $\mathbf{3 0}$ cases.

\section{TERMINOLOGY}

In this paper the elements of the cauda equina are referred to as filia. From the point at which these evaginate the dural sheath around the cord to the point of fusion of the dorsal and ventral components distal to the ganglion, they are called roots. More distally again they are termed nerves, at first forming part of the lumbo-sacral plexus. The lumbosacral cord is that segment of the anterior primary division of the fifth lumbar nerve extending from the

${ }^{1}$ Present address: Pathology Department, Metropolitan General Hospital, Cleveland, Ohio, U.S.A. point at which it receives a contribution from the fourth lumbar nerve until its junction with the first sacral nerve, or that part of the fifth lumbar nerve which runs over the ala of the sacrum.

\section{MATERIAL AND METHODS}

Cadavers were selected from routine necropsies as time and circumstance permitted. Although an attempt was made to obtain these as soon as possible after death and before refrigeration, a wide range of body temperatures was encountered, varying from warm fresh bodies to others which were very cold. Rigor mortis was present in varying degrees in most of these and had to be broken down by forcible ventroflexion of the hips and dorsiflexion of the knees. The ankles were not mobilized and were left at approximately 90 degrees to the leg.

To retain anatomical stability and avoid loosening of the spinal dura, filia and nerve roots which, in the lumbosacral region, run with a ventral convexity in the spinal canal, and to afford exposure of the nerves running over the ala of the sacrum, a technique of anterior dissection with minimal interference to neural structures was devised. After removal of abdominal viscera, the right lumbo-sacral nerves were exposed by dissection of the anterior part of the psoas muscle and overlying fatty tissue and fascia. Care was taken not to clean the nerves and, so far as possible, to avoid breaking down any fibrous adhesions present. The amount of movement was than observed at selected points outside the intervertebral foramina during straight leg raising. The right halves of the vertebral bodies of L3 to L5 were next removed by cutting through their pedicles with a vibratory saw as far anteriorly as possible and by a second line of section along the mid-sagittal plane of the vertebral bodies. The upper part of the sacrum was removed by an oblique cut beginning just medial to the lumbo-sacral cord and first sacral foramen, joining this with an extension of the mid-line saw cut. Finally, a central longitudinal incision was made on the ventral surface of the exposed dura to reveal the elements of the cauda equina. Straight leg raising was then repeated. In a series of $\mathbf{3 0}$ consecutive dissections restricted to subjects without known or obvious spinal deformity, the amounts of movement were measured. These included 21 male and nine female subjects of a wide age range. The points at 


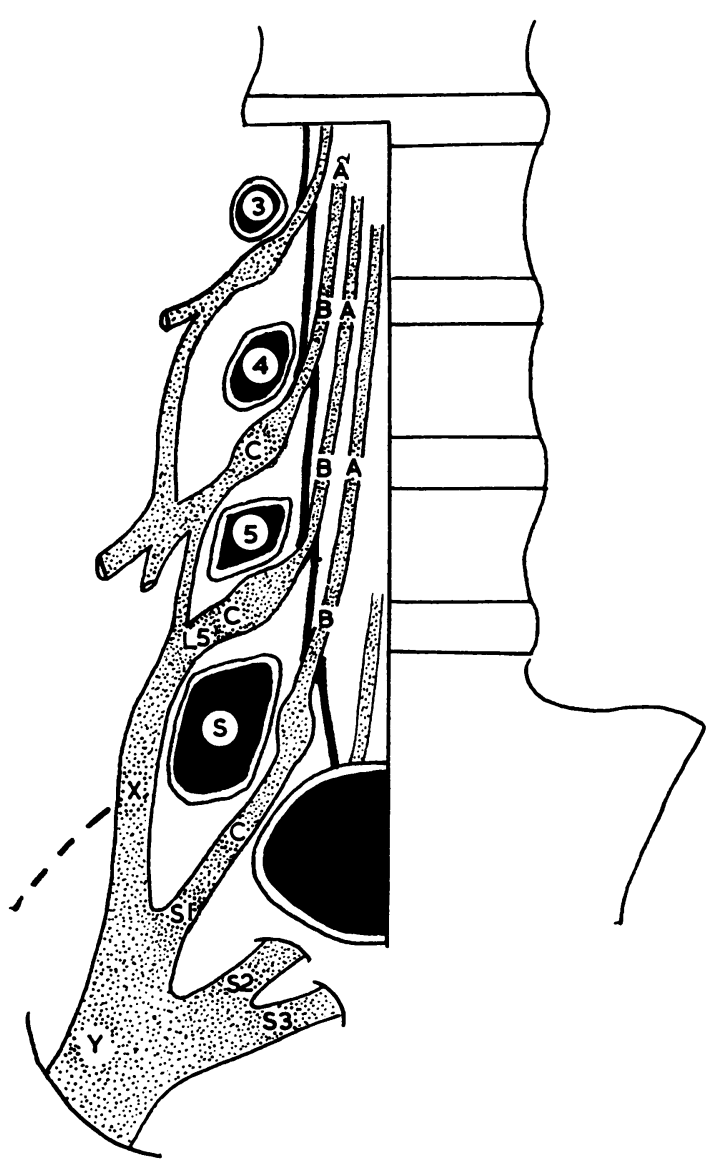

FIG. 1. The dissection as seen from the ventral aspect, showing the points at which measurements were made.

which measurement were made on the L4, L5, and S1 nerves are indicated in Figure 1. Points $A$ and $B$ were located on the filia, point $B$ being situated just above the formation of the nerve root and point $A$ being one segment cephalad. Point $C$ was on the nerve in the intervertebral or sacral foramen.

$\mathrm{X}$ lay on the lumbo-sacral cord as it passed over the ala of the sacrum and $Y$ was on the sciatic nerve approximately $1 \mathrm{~cm}$. proximal to the sciatic notch. Measurements were made to the nearest millimetre only, using metal pins inserted into the perineurium and paper markers placed on the adjoining bone (Fig. 2). On other occasions a marked metal necropsy needle was held along the line of curvature of the nerve and measurements were made against suitable natural landmarks such as small blood vessels in the nerve sheath. The wide range of variation found in different cadavers of similar ages did not appear to make any highly accurate attempts at mensuration justifiable. This anterior method of dissection precluded any critical examination of the intervertebral discs for

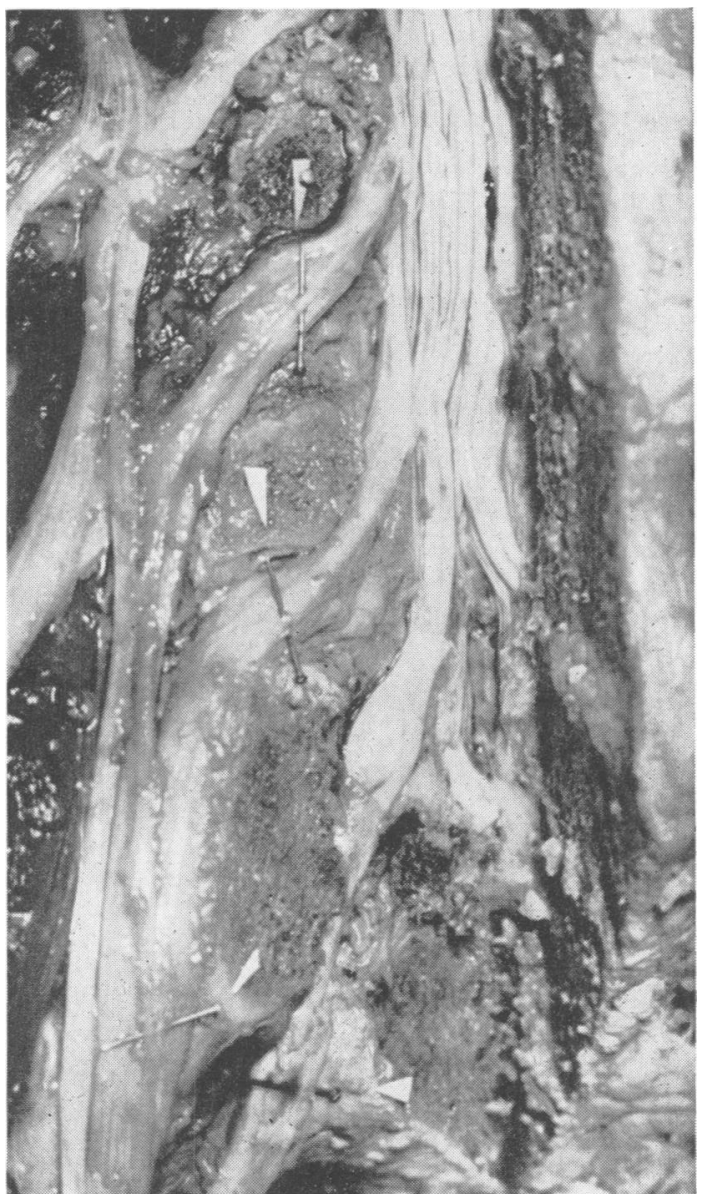

FIG. 2. Pins have been inserted through the perineurium L4, L5, S1, and the lumbo-sacral cord as it passes over the ala of the sacrum. Paper markers have been placed on the transected vertebral pedicles. The filia of the cauda equina are seen inside the opened dura.

prolapse of the nucleus pulposus. However, in quite a number of dissections disordered fibrous tissue was found adherent to the nerves in the region of the L5 intervertebral foramen and suggested previous rupture or disintegration of the disc structure.

\section{RESULTS}

Refrigeration appeared to cause no impairment of downward movement of nerves on straight leg raising but it was noted that in the coldest cadavers the time taken by nerves and roots to recoil to their previous positions was markedly increased. The range and average amount of movement at different points in the sciatic nerve and lumbo-sacral nerve roots as found by measurement is given in the Table for 


\section{TABLE}

AVERAGE MOVEMENTS ${ }^{1}$ AT DIFFERENT POINTS ${ }^{2}$ ON THE COURSE OF FOURTH AND FIFTH LUMBAR, FIRST SACRAL, AND SCIATIC NERVES ON STRAIGHT LEG RAISING

\begin{tabular}{cllll} 
Age Group & $<35 y r$. & $36-55 y r$. & $56-75 y r$. & $>75 y r$. \\
\hline No. of cases & 8 & 9 & 9 & 4 \\
L4 A & 0 & 0 & 0 & 0 \\
& $(0)$ & $(0)$ & $(0)$ & $(0)$ \\
B & 1 & $0 \cdot 5$ & 0 & 0 \\
& $(0-1)$ & $(0-1)$ & $(0)$ & $(0)$ \\
C & 2 & $1 \cdot 5$ & $0 \cdot 5$ & 0 \\
L5 A & $(0-4)$ & $(0-3)$ & $(0-1)$ & $(0-1)$ \\
& 1 & $0 \cdot 5$ & 0 & 0 \\
B & $(0-2)$ & $(0-1)$ & $(0)$ & $(0)$ \\
& 2 & 1 & 0 & 0 \\
C & $(1-4)$ & $(0-2)$ & $(0-1)$ & $(0)$ \\
& 3 & 3 & $1 \cdot 5$ & $1 \cdot 5$ \\
S1 A & $(1-6)$ & $(1-5)$ & $(0-3)$ & $(1-2)$ \\
& 1 & 1 & 0 & 0 \\
B & $(0-2)$ & $(0-2)$ & $(0)$ & $(0)$ \\
& 3 & 2 & 1 & $0 \cdot 5$ \\
C & $(0-4)$ & $(0-4)$ & $(0-4)$ & $(0-1)$ \\
Sciatic nerve & 5 & 4 & 3 & 2 \\
X & $(3-7)$ & $(2-7)$ & $(1-6)$ & $(1-4)$ \\
Y & $4 \cdot 5$ & $4 \cdot 5$ & 3 & $2 \cdot 5$ \\
Y & $(3-7)$ & $(2-7)$ & $(1-5)$ & $(2-3)$ \\
& $7 \cdot 5$ & $6 \cdot 5$ & $4 \cdot 5$ & 4 \\
& $(6-10)$ & $(3-10)$ & $(3-8)$ & $(4-5)$
\end{tabular}

${ }^{1}$ The measurements are recorded in millimetres. The range of movements recorded at the same point in different cadavers of the same age group is given in brackets.

${ }^{2}$ The points are shown in Fig. 1 and described in the text.

different age groups, and is illustrated in Figures 3, 4 , and 5. No downward movement of the third lumbar nerve was seen in any case. The amount of displacement of the fourth lumbar nerve at its foramen and, more proximally, in its roots or intradural filia appeared to depend on the degree to which it entered into the formation of the lumbosacral cord and thus formed part of the sciatic nerve. Where the L4 component of the sciatic nerve was large, greater movement was seen. This branch was absent in one case, noticeably small in a few others, and quite large in the remainder. Movements in the root of the fifth lumbar nerve were of the order of $3 \mathbf{m m}$. in younger subjects. The greatest amount of movement was found in the first sacral nerve, averaging 4 to $5 \mathrm{~mm}$. Measurements of the $S 2$ and $S 3$ nerves and roots were not made, but to visual observation these appeared to move to much the same degree as S1. The Table shows clearly that the greatest amount of movement induced by straight leg raising occurred distally in the sciatic nerve. Only a relatively small amount of movement was transmitted to the intradural filia, and this movement in particular was absent in the oldest age groups. Even this shift when present was always absorbed within the distance of one or two vertebrae and at no time could it be said that the cauda equina was placed under tension. It can also be seen that the amount of movement at all points examined decreased with increasing age. In most instances movement in the sciatic nerve at its exit from the pelvis (point $\mathrm{Y}$ ) commenced when the heel was elevated only 1 to 3 inches from a horizontal position. This movement was transmitted proximally in the nerve as elevation continued and almost immediately affected the lumbo-sacral cord as it passed over the ala of the sacrum. At the intervertebral foramina, movements could only be noticed when an elevation of some 20 to 30 degrees was achieved. Little additional movement at any point could be observed after 70 degrees of straight leg raising.

ANATOMICAL COURSE, PRESSURE POINTS, AND TENSION

Figure 2 shows clearly that both L4 and L5 run a sigmoid course through the intervertebral foramina, impinging first on the infero-medial aspect of the upper pedicle and then on the supero-lateral surface of the pedicle below. The lumbo-sacral cord runs over a marked ventral convexity at the ala of the sacrum (Fig. 3), and then downwards, backwards, and laterally to the sciatic notch. S1 runs in more direct fashion, curving gradually backwards ando outwards, whereas $S 2$ and $S 3$ again curve in more marked manner through their openings in the anterior surface of the sacrum. The sciatic nerve is $\vec{B}$ formed on the antero-medial surface of the pirie T) formis muscle and then passes slightly laterally below the muscle and through the greater sciatif foramen, lying for some distance in direct contace with the dorsal aspect of the ischium. It then passes over the dorsal surface of the superior gemellus, the obturator internis, the inferior gemellus, and the quadratus femoris muscles. With straight leg raising the nerve is first drawn straight downwards through the notch and then it moves antero-laterally. It becomes tightly apposed to the underlying bone and the degree of pressure produced here is quite remarkable. Similar pressure occurs between the lumbo-sacral cord and the ala of the sacrum, and to lesser degrees at both the L4 and L5 intervertebral foramina, between the nerves and the vertebral pedicles.

\section{ADHESIONS}

Throughout their lumbo-pelvic course the sciatic nerve and its constituent roots are lightly tethered by innumerable filmy fibrous strands to adjoining fascia and periosteum. At the foramina these adhesions extend also onto the lateral extension of the posterior common spinal ligament. If the nerve roots $N$ are dissected free more downward movement can be obtained on straight leg elevation, but the degree of natural recoil is affected. In younger subjects the 


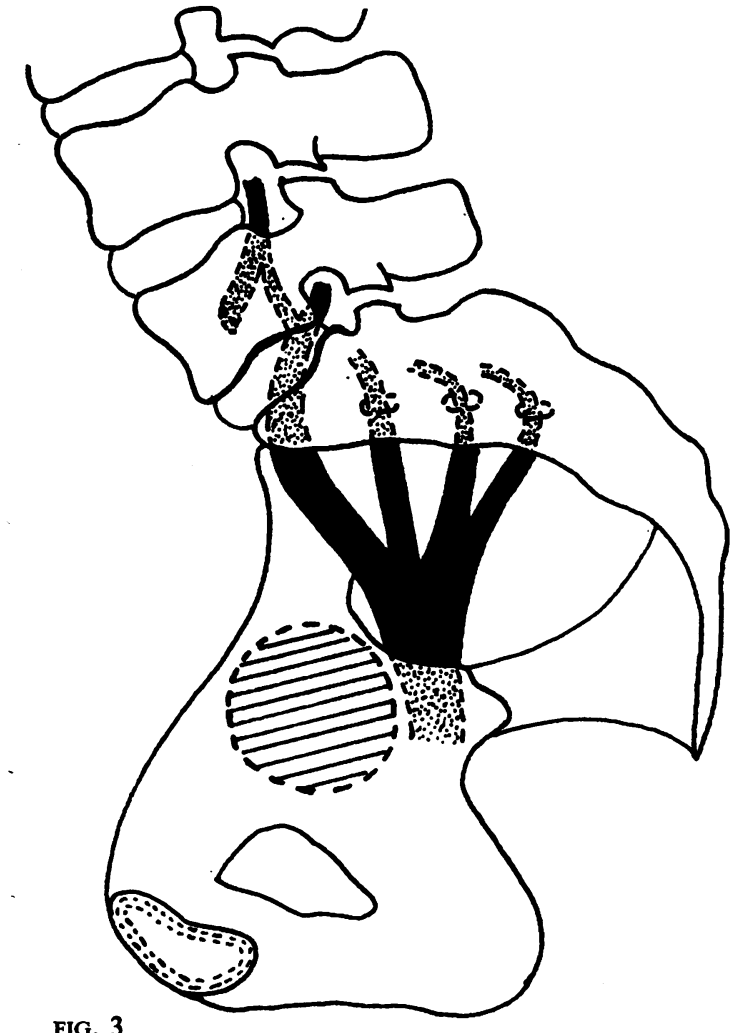

FIG. 3

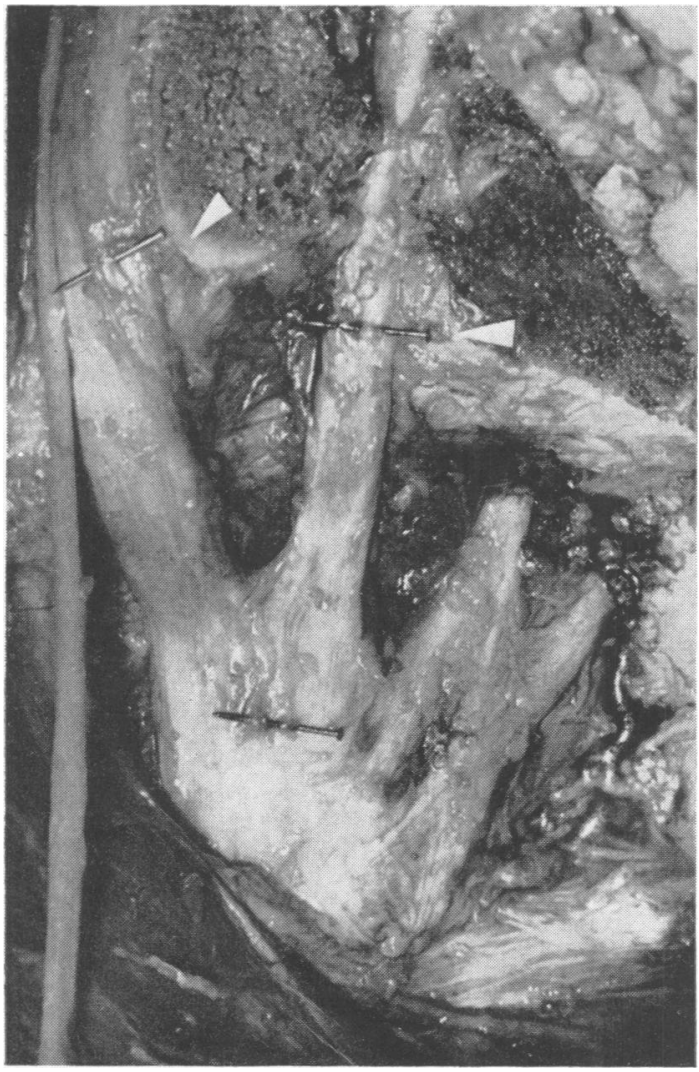

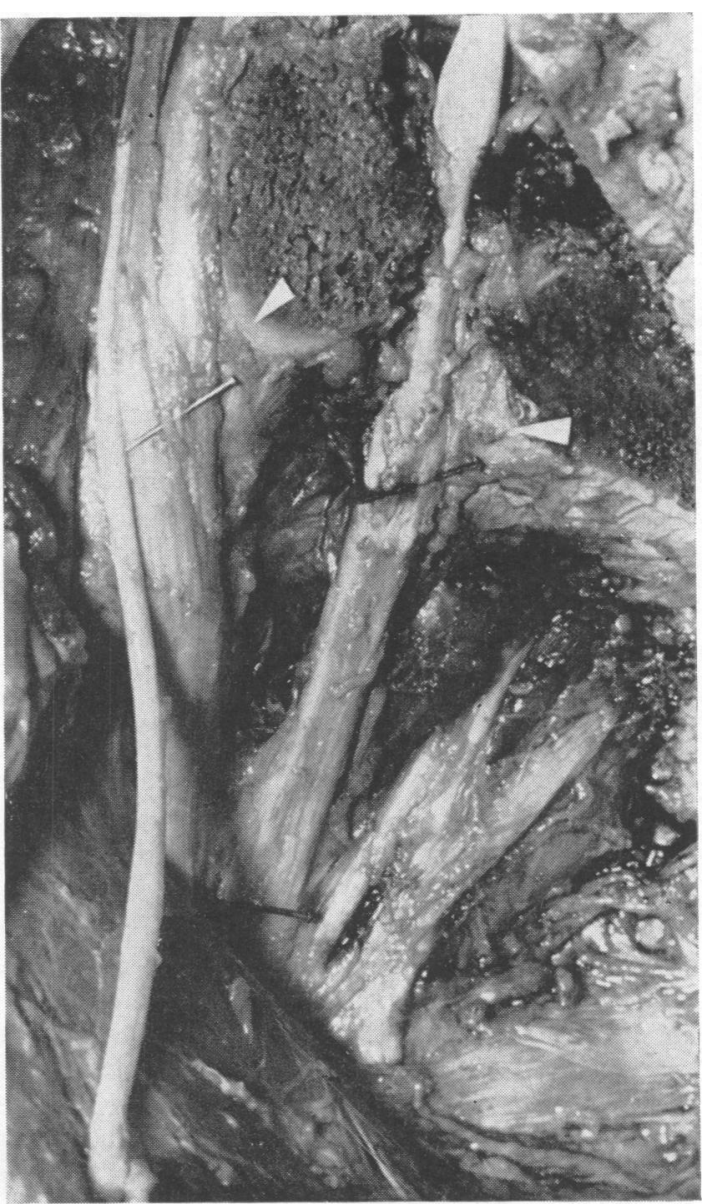

FIG. 5

FIG. 3. Diagram of lumbo-sacral nerves and sciatic nerve from medial aspect, showing the tortuous course of the nerves apart from $S 1$, which descends in a relatively straight line.

FIG. 4. The sciatic nerve inside the pelvis before straight leg raising. Pins have been inserted into the lumbo-sacral cord, $S 1$, and the sciatic nerve.

FIG. 5. The sciatic nerve inside the pelvis during straight leg raising. The pins have moved distally relative to paper markers and the sciatic notch. 
fourth and fifth lumbar nerves, if divided at the ala of the sacrum, can easily be stripped out of their beds in the foramina. With advancing age the density of adhesions in this region is often noticeably increased. In several cases there were dense adhesions to fibrous masses derived from the outer part of the disc and old prolapses seemed possible. In these, attempts to strip nerves out of their foramina were futile without sharp dissection. In three cadavers, dense adhesions were found between the lumbosacral nerves and periosteum at points considerably removed from the spinal canal. In each of the two more recent cases, a convincing clinical diagnosis of sciatica had been made. The first of these (coroner's necropsy) was a 36-year-old white male who clearly had dense fibrous adhesions between nerves and periosteum at the first anterior sacral foramen and again at the ala of the sacrum, extending proximally around the fifth lumbar nerve into the outer part of the intervertebral foramen. No prolapse of disc was noted. No neurological history was known until later, when the general practitioner volunteered the information that this man had been suffering from sciatica, with lumbo-sacral backache and pain radiating down the right leg, for some months before death. The other case was a 44-year-old female, admitted with severe back pain and limitation of straight leg raising to 50 degrees. The clinical diagnosis was sciatica. Death was unexpected and necropsy revealed a small adenocarcinoma of the stomach with a dense sheet of metastatic malignant tissue spreading diffusely over the anterior surface of the sacrum and firmly anchoring the sacral nerves at their foramina. No other nerves were involved. Straight leg raising was seen to increase tension in the sciatic nerve without any transmission through the sacral foramina to the canal. No tumour was found in the canal itself.

\section{DISCUSSION}

MOVEMENT The amount of movement found at the intervertebral foramina is in general accord with that previously recorded by Inman and Saunders (1942), by Falconer, McGeorge, and Begg (1948), and by Charnley (1951). The relatively large shift at the sciatic notch and over the ala of the sacrum (Figs. 4 and 5) does not appear to have been appreciated previously. The statement by Charnley (1951), that the sciatic nerve behaves as if devoid of elasticity, cannot be accepted, and indeed the reason why movement becomes progressively less towards the foramina appears to be largely due to the natural elastic properties of the nerves. The tethering effect of innumerable filmy fibrous adhesions, and particularly those in the region of the foramina, makes the nerve root a relatively fixed point from which downward stretch occurs. This fixity appears to be directly due and proportionate to the density of adhesions present, and these in turn appear to be related to age, and to changes caused by wear and tear. In youth considerable to-and-fro movements may be seen, but where the structure of the disc has been disrupted, adhesions may prevent all movement. It is our firm impression that in those cases where downward movement is less, tension in the nerve and pressure over the bony prominences are correspondingly increased.

ANATOMICAL COURSE The description of the sciatic nerve as emerging from the vertebral foramina and descending in an almost straight line through the pelvis posterior to the hip joint (Charnley, 1951) is a considerable over-simplification. Viewed from the lateral aspect, the diagram given by Cram (1953) correctly illustrates the curvatures of the lumbar nerves in the antero-posterior plane, including the curve over the ala of the sacrum. The second and third sacral roots run backwards due to the fact that they must follow the direction of the sacral canalo Only the first sacral nerve has anything like a direce course downwards and this curves gently laterally? (Figs. 1 and 2). Most diagrams illustrating the lumbo® sacral nerve roots depict them as running obliquel but in a straight line outwards through their foramina (Begg, Falconer, and McGeorge, 1946; Falconeo et al., 1948; Armstrong, 1952). Inman and Saunders (1942 and 1947) state that the nerve occupies $\mathscr{Q}$ only the upper half of its foramen. In fact, the or fourth and fifth roots impinge first on the inferomedial aspect of the pedicle and then as nerves on the superior and lateral aspects of the pedicle below, crossing the foramen obliquely and coming into relation with the disc in their lower and outer part. The course of the fifth nerve in particular is definitely sigmoid (Figs. 1 and 3). Forced lessening of the lumbar lordosis at necropsy by pressure on the anterior surfaces of the lumbar vertebrae results in slight separation of the pedicles and widening of the intervertebral foraminal space. This allows the roots to run a more direct course outwards and appears to cause some slackening. This observation may have some bearing on the cause of the flattening of the lumbar lordosis seen in certain cases of disc protrusion, which has been extensively discussed by Charnley (1951). It has been argued by O'Connell (1943) that such flattening must increase the length $\frac{D}{O}$ of the spinal canal and thus induce tension in the dura and roots. While it may be accepted that there is $N$ slight lengthening of the canal on ventroflexion of the spine, the critical question is whether any in- 0 crease in dural tension is accompanied by sufficient $\mathrm{\omega}$ 
upward movements to maintain or increase the pull on a root which is simultaneously being slackened by separation of the pedicles. From our observations it seems probable that there is a nett gain in favour of the root, in that loss of lumbar lordosis may be a mechanism affording relief from root pressure in direct fashion and without involving the sciatic nerve as such. Very small changes appear to be critical here as can be shown by the well-known induction of sciatic pain by flexion of the neck. Such movements produce only minor shifts in the lumbar dura.

STRAIGHT LEG RAISING The fact that straight leg raising can cause large movements in the sciatic nerve, and very considerable compression of the nerve against the bone without resulting in symptoms, raises the question of the mechanism whereby prolapsed discs induce pain. Compression by discs has by itself been considered inadequate (O'Connell, 1943; Inman and Saunders, 1947; Charnley, 1951; Breig and Marions, 1962). Tension has been advocated, but again this by itself would appear to have no effect. O'Connell (1943) has suggested that the root may be regarded as being held between the dura proximally and the limb plexus distally, and that tension may be induced by a disc prolapse. Something more definite in the way of attachments must be envisaged, however, particularly in young people. Dense adhesions, in our experience, are common and could readily be invoked.

Inflammation has been suggested as a common precipitating factor. Although the concept of an interstitial sciatic neuritis is currently in discard, occasional reports of a radiculitis without prolapse can be found (Holmes and Sworn, 1945). In the presence of disc protrusions, Begg et al. (1946) and Falconer et al. (1948) have reported congestion and swelling of the filia, and these authors consider that the production of sciatic symptoms depends on two factors, disc prolapse and oedematous changes within the root. Histological evidence in this respect is scanty, but there is not infrequent objective necropsy evidence of extensive fibrosis between nerves and bones, particularly in the presence of disorganized discs. This is seen particularly at the foramina and may also be present over the ala of the sacrum.

The fact that stretch and movement of the sciatic nerve is first observable at the sciatic notch and only later at the roots has some implications in interpretation of the straight-leg-raising test. If patients with sciatica develop pain immediately or shortly after beginning this manoeuvre, it may imply a sensitive nerve at the sciatic notch or at the ala of the sacrum. There are many clinical reports of tenderness throughout the course of the sciatic nerve which suggest that an undue sensitivity can be present over quite extensive lengths. In all probability this is due to intrinsic changes. Charnley (1951) has made a clinical division of cases of sciatica into those with and those without severe limitation of straight leg raising. The former almost always have protruded discs and respond well to surgery.

If straight leg raising does not induce pain two possibilities arise. Movement may not be transmitted to the root, and fibrous adhesions as described could well cause this. Alternatively, nerve roots may be affected by some intrinsic rather than extrinsic lesion. Here it may be noted that Charnley (1951) found that those patients with only slight limitation of straight leg raising seldom had protruded discs.

When the straight-leg-raising test is combined with an examination of ability to perform forward flexion, multiple different forces come into play on the nerves and cord. Flexion of the spine tends to draw the nerves upwards (Breig and Marions, 1962) but simultaneously shortens the course followed by the roots and lessens the dorsal protrusion of some disc prolapses (Begg et al., 1946). Straight leg raising pulls the roots and dura downwards, and, depending on the tenseness of the hamstring muscles, may tilt the pelvis. The unpredictable factor is how much these movements may be modified by the existence of adhesions between the nerve and bone, and the variable results of combined tests of flexion and straight leg raising (Charnley, 1951) may be explicable on these anatomical considerations rather than as psychological phenomena.

\section{SUMMARY}

An anterior dissection of the lumbo-sacral region, as performed in 30 cadavers of widely varying ages, is described.

The amount of movement of the sciatic nerve, the lumbo-sacral nerve roots, and the filia of the cauda equina, which was produced by straight leg raising, was measured. All movements were found to decrease with advancing age and with proximity to the spinal cord. Pressure points and adhesions between the nerves and periosteum are described, and their significance in the causation of sciatica is discussed.

\section{REFERENCES}

Armstrong, J. R. (1952). Lumbar Disc Lesions. Livingstone, Edinburgh and London.

Begg, A. C., Falconer, M. A., and McGeorge, M. (1946). Myelography in lumbar intervertebral disk lesions. A correlation with operative findings. Brit. J. Surg., 34, 141-157.

Breig, A., and Marions, O. (1962). Biomechanics of the lumbo-sacral nerve roots. Personal communication.

Charnley, J. (1951). Orthopaedic signs in the diagnosis of disc protrusion. Lancet. 1 186-192 
Cram, R. H. (1953). A sign of sciatic nerve root pressure. J. Bone J. Surg., 35 1 , 192-195.

Falconer, M. A., McGeorge, M., and Begg, A. C. (1948). Observations on the cause and mechanism of symptom-production in sciatica and low-back pain. J. Neurol. Neurosurg. Psychiat., 11, 1326.

Holmes, J. M., and Sworn, B. R. (1945). Sciatic 'neuritis'. Brit. med. J., 2, 350-351.
Inman, V. T., and Saunders, J. B. de C. M. (1942). The clinico-anatomical aspects of the lumbosacral region. Radiology, 38, 669-678.

7 , (1947). Anatomico-physiological aspects of injuries to the intervertebral disc. J. Bone J. Surg., 29, 461-468.

O'Connell, J. E. A. (1943). Sciatica and the mechanism of the production of the clinical syndrome in protrusions of the lumbar intervertebral discs. Brit. J. Surg., 30, 315-327 